\title{
Duration of breastfeeding is associated with emotional eating through its effect on alexithymia in boys, but not girls
}

\author{
Tatjana van Strien $^{\mathrm{a}, \mathrm{b}, *}$, Roseriet Beijers ${ }^{\mathrm{a}}$, Sanny Smeekens ${ }^{\mathrm{c}}$, Laura H.H. Winkens ${ }^{\mathrm{b}}$ \\ a Radboud University Nijmegen, Behavioural Science Institute, Nijmegen, the Netherlands \\ ${ }^{\mathrm{b}}$ Department of Health Sciences, Faculty of Science, Vrije Universiteit Amsterdam, Amsterdam Public Health Research Institute, Amsterdam, the Netherlands \\ ${ }^{\mathrm{c}}$ Faculty of Psychology and Educational Sciences, Open University of the Netherlands, the Netherlands
}

\section{A R T I C L E I N F O}

\section{Keywords:}

Breastfeeding

Emotional eating

Alexithymia

Longitudinal

Sex-differences

\begin{abstract}
A B S T R A C T
Emotional eating (EE), or eating in response to negative emotions, was earlier shown to be associated with difficulty in identifying emotions (alexithymia). To improve our understanding of possible causes of alexithymia and EE, we assessed possible associations with duration of breastfeeding in infancy. The aim of the present study was to examine in a prospective, longitudinal study whether duration of breastfeeding is associated with EE in adolescence, through its effect on alexithymia difficulty identifying emotions, and whether this mediation effect is contingent on gender. Our hypothesis was that longer duration of breastfeeding would be associated with lower EE in adolescence through its effect on lower alexithymia difficulty identifying feelings in boys but not in girls (Moderated mediation). The sample included 129 children and their families (67 boys and 62 girls). Duration of breastfeeding was reported by the mother when the infant was 15 months old. Alexithymia difficulty identifying feelings (Toronto Alexithymia Scale) and EE (Dutch Eating Behavior Questionnaire) were reported by the child at 12 years of age. EE was also reported by the child at 16 years of age. Moderated mediation was significant for EE at 12 years, and borderline significant for EE at 16 years. As hypothesized, for boys but not for girls, longer duration of breastfeeding was related to less difficulties in identifying feelings, resulting in lower degrees of EE in adolescence. It is concluded that breastfeeding in infancy may protect boys against EE through its positive association with better ability to identify feelings.
\end{abstract}

\section{Introduction}

Emotional eating (EE), defined as eating in response to negative emotions (Van Strien, Donker, \& Ouwens, 2016), is associated with prospective weight gain (Koenders \& Van Strien, 2011; Van Strien, Herman, \& Verheijden, 2012), binge eating in adult (clinical) samples (Klump et al., 2014; Van Strien, Engels, Van Leeuwe, \& Snoek, 2005), and acts as a mediator between depression and weight gain (Van Strien, Konttinen, Homberg, Engels, \& Winkens, 2016; Vittengl, 2018). EE is an a-typical stress response. The typical stress response is not eating, because the physiological stress reactions mimic the internal sensations associated with feeding induced satiety (Gold \& Chrousos, 2002). It has been postulated that the a-typical stress response of EE develops early in life (Wardle, 1990), as a possible outcome of parental feeding practices (Carper, Fisher, \& Birch, 2000; Van Stien and Bazelier, 2007; Van Strien, Van Niekerk, \& Ouwens, 2009) and more general parenting practices where there was insufficient regard for the child's needs
(Bruch, 1973; Snoek, Engels, Janssens, \& Van Strien, 2007; Escobar et al., 2014; Zhu, Luo, Cai, Li \& Liu, 2014). If parental responses to the child's needs are continuously inappropriate, be it neglectful, indiscriminately permissive or over-controlling, the child may develop i) poor satiety awareness (poor awareness of physiological symptoms associated with hunger and satiety) and ii) poor emotional awareness (poor awareness of emotions, resulting in difficulty identifying and describing emotions, also referred to as alexithymia ${ }^{1}$ ) (Garner, 1991). Poor satiety awareness and alexithymia were indeed shown to be associated with higher EE (Larsen, van Strien, Eisinga, \& Engels, 2006; Van Strien et al., 2005; Van Strien \& Ouwens, 2007). The present study focusses on the feeding practice of breastfeeding. As will be elaborated below, there is ample evidence that breastfeeding is associated with satiety awareness, and that breastfeeding may be associated with EE through its impact on satiety awareness. In contrast, there is only circumstantial evidence that breastfeeding may also be associated with alexithymia. Therefore, the present study aims to examine whether

\footnotetext{
* Corresponding author. Radboud University Nijmegen, Behavioural Science Institute, Nijmegen, the Netherlands.

E-mail address: t.vanstrien@psych.ru.nl (T. van Strien).

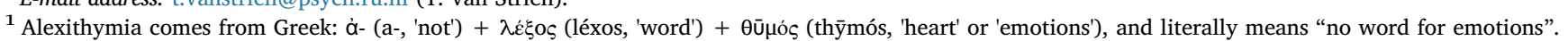


duration of breastfeeding is associated with EE in adolescence, through its effect on alexithymia.

\subsection{Breastfeeding and satiety awareness}

There is ample evidence that duration of breastfeeding is associated with satiety awareness. For example, when compared to formula fed infants, breastfed infants are rated by their mothers to have a better satiety awareness and appetite regulation (Brown \& Lee, 2012; Disantis, Collins, Fisher, \& Davey, 2011; Reyes et al., 2014). Several biological constituents present in breast milk, but absent in infant formula, could be responsible for the better satiety awareness of breastfed infants. For example, breast milk contains many hormones, including leptin. Leptin, also referred to as the satiety hormone, helps to regulate the energy balance by inhibiting hunger. A decreased sensitivity to leptin results in an inability to detect satiety, and has been related to obesity and emotional eating (Michels, Sioen, Ruige, \& De Henauw, 2017; Schwartz, Woods, Porte, Seeley, \& Baskin, 2000). Furthermore, breast milk changes in energy density and fat content during the day (Nommsen, Lovelady, Heinig, Lönnerdal, \& Dewey, 1991). Breastfed infants learn to respond to this variation and to match their own intake to their needs (Brown \& Lee, 2012).

\subsection{Breastfeeding and alexithymia}

In contrast to ample evidence on the positive association of breastfeeding with a better awareness of satiety, only circumstantial evidence exists on the possible association of breastfeeding with awareness of emotions (alexithymia). A link between breastfeeding and alexithymia could be hypothesized on the following grounds.

First, breastfeeding could be a protective factor for later alexithymia and emotional eating, because breastfeeding is regarded as a more responsive style of feeding (Shloim, Shafiq, Blundell-Birtill, \& Hetherington, 2018; Shloim, Vereijken, Blundell, \& Hetherington, 2017). When breastfeeding, the mother has to rely on the feeding signals of the infant (e.g. turning head away), rather than on external cues such as the quantity of milk left in the bottle (Woo \& Martin, 2015). Next to increased responsiveness during the lactation period, breastfeeding was shown to set the stage for less controlling and more responsive parental feeding and parenting practices later in childhood (Brown \& Arnott, 2014; Jansen, Mallan, Byrne, Diet, Daniels, \& Nicholson, 2016; Taveras, Scanlon, Bearch, Rifas-Shiman, RichÉdwards, \& Gillman, 2004; Ventura, 2017; Weaver, Schofield, \& Papp, 2018). For example, a recent study using data from the large NICHD study found that longer breastfeeding duration predicted increases in observed maternal sensitivity up to child age 11 years (Weaver et al., 2018). In turn, more sensitive and cooperative parenting is associated with lower degrees of the alexithymia aspects difficulties identifying emotions and difficulties describing emotions (see the meta-analysis of Thorberg, Young, Sullivan, \& Lyvers, 2011).

Second, unlike formula milk, breast milk contains small amounts of hormones, (Uvnas-Moberg, 1998). Accumulating evidence suggests that hormones from the mother ingested through milk, affect the offspring's own hormonal concentrations and developing brain (Hinde et al., 2014). Breast milk also includes the hormone oxytocin (OT). OT has been shown to facilitate socio-emotional abilities, including sensitivity and attunement to emotional expressions of others. This sensitivity and attunement is impaired in people with high degrees of alexithymia (Grynberg et al., 2012), possibly because awareness of others' emotions is related to awareness of one's own emotions. It can thus by hypothesized that increased exposure to oxytocin, through longer breastfeeding duration, predicts higher sensitivity to emotional expressions of others and lower degrees of alexithymia (Luminet, Grynberg, Ruzette, \& Mikolajczak, 2011). Research supports this hypothetical mechanism. For example, the social impairment of men with high degrees of alexithymia could be remedied by intranasal

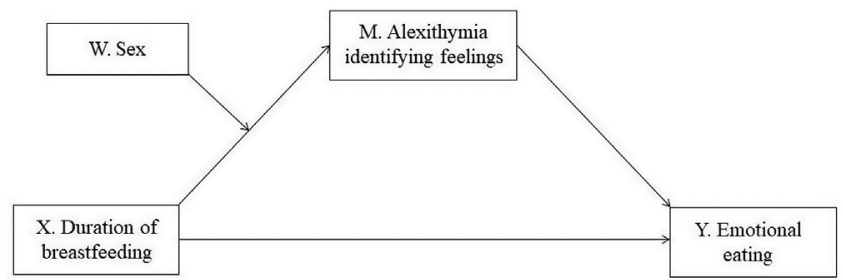

Fig. 1. Conceptual diagram of moderated mediation: moderation of sex $(W)$ on the mediation effect of alexithymia identifying feelings $(\mathrm{M})$ between duration of breastfeeding (X) and EE (Y).

administration of oxytocin (Luminet et al., 2011). Moreover, Krol, Rajhans, Missina, and Grossmann (2015a) found that exclusive breastfeeding duration was positively associated with attention to emotional bodily and facial expressions from others. The hypothesis that oxytocin is the underlying mechanism of this association was supported in a subsequent study (Krol, Monakhov, San Lai, Ebstein, \& Grossmann, 2015b). In this study, the effect of exclusive breastfeeding on attention to emotional expressions was shown to be most pronounced in infants with a genotype that was associated with a decreased availability of oxytocin (Krol et al., 2015b), perhaps because the small quantities of oxytocin in breast milk act "as a transcriptional promotor of the oxytocin gene" (Shafai, Mustafa, Mulari, \& Curtis, 2017, p. 80). To our knowledge, however, the hypothesis that shorter duration of breastfeeding is associated with higher degrees of alexithymia, and subsequently with more EE in adolescence, has not been investigated.

\subsection{Differential sex effects}

The mediation effect of alexithymia in the association between breastfeeding duration and EE may be moderated by sex (see Fig. 1 for the model of moderated mediation). Breastfeeding may particularly protect boys against high degrees of alexithymia. Evidence suggests that effects of parenting are sex-specific, as particularly boys seem to be protected against emotional disorders by responsive parenting (Browne, Odueyungbo, Thabane, Byrne, \& Smart, 2010). Moreover, the effects of oxytocin on social sensitivity and awareness of emotions have also been shown to be sex specific, with no, or even opposite, effects during social interaction in women. That is, whereas social interactions showed improvement in men, the interactions deteriorated in women after oxytocin administration (e.g. Rilling et al., 2014). Combined with the lower capacity for empathy (Christov-Moore et al., 2014) and the higher prevalence of alexithymia in men (Levant, Hall, Williams, \& Hasan, 2009), we hypothesized that the mediation effect only holds true for boys.

\subsection{Present study}

Taken together, breastfeeding duration may be associated with lower alexithymia because: 1 ) breastfeeding is associated with a more responsive style of parenting, which, in turn, is associated with lower degrees of alexithymia, and 2) the oxytocin present in breast milk may contribute to a better ability to identify emotions of others, which may be related to a better ability to also identify one owns emotions. Together with evidence that alexithymia is positively associated with EE (e.g. Larsen et al., 2006), we expected alexithymia to be a mediator between breastfeeding duration and EE. A longer duration of breastfeeding is hypothesized to be associated with less emotional eating through a better ability to identify feelings, compared to a shorter duration of breastfeeding. We focused our hypothesis on the alexithymia aspect identifying feelings, as we suggest that breastfeeding has an impact on this specific alexithymia aspect and not the alexithymia aspect describing feelings. We further expected this hypothesis to be 
only true for boys, not girls.

We tested our hypothesis in a prospective longitudinal study. Duration of breastfeeding was reported by the mother when the child was 15 months old. Alexithymia and EE were reported by the child at the age of 12 years. EE was also assessed at 16 years by child self-report. This enabled us to improve our test of the causal chain, because not only the predictor but also the mediator preceded emotional eating in real time. In order to examine whether the moderated mediation model is specific for EE, we also assessed the effects of breastfeeding through alexithymia on external eating (eating in response to external food cues such as smell and sight of food). External eating was reported by the child at 12 years and 16 years of age.

\section{Method}

\subsection{Participants}

The research questions of this study were addressed using data from the Nijmegen Longitudinal Study on Infant and Child Development. This study started in 1998 with a community sample of 129 healthy 15month-old children ( $52 \%$ boys) and their primary caregivers. For the Nijmegen Longitudinal Study on Infant and Child Development, the following IRB approvals had been obtained: ECSW20213-1811-157 and ECG2013-1308-123/feature: COM/MB/13U.016212. The families were recruited on the basis of records from local health-care centers in the city of Nijmegen (The Netherlands). Families with a 15-month-old baby were sent an invitation to participate in the study that aimed to gain more insight into children's development in the first years of life. If they met the two eligibility criteria (i.e., sufficient fluency in Dutch and child without serious health problems) and were interested in participation, they could return a response card. Of the 639 families approached, 174 replied and 129 families were randomly selected for the study (129 families was the maximum attainable given the time and resources available for the project at that moment). As indicated by the distribution of parental and child measures at 15 months, the initial sample was representative of the Dutch population of families with young children (Van Bakel \& Riksen-Walraven, 2002a, 2002b). In 126 families, the mother was the primary caregiver and in the remainder of the families this was the father. The primary caregivers were between 22 and 47 years of age at the time of the recruitment.

Of the original sample of 129 children, 111 children participated at age $12\left(M_{\text {age }}=12.38\right.$ years, $S D=0.28 ; 52 \%$ boys $)$ and 101 children at age $16\left(M_{\text {age }}=16.25\right.$ years, $S D=0.30 ; 50 \%$ boys $)$. See Fig. 2 for an overview of the recruitment process and drop-out of the study. To determine whether there was selective drop-out or not, the participating families in the 12- and 16-year assessment were compared to the families that did not take part on the study variables included in the present study (i.e., duration of breastfeeding, and paternal and maternal level of education). Independent $t$-tests revealed no significant differences. Of the children who participated, 104 children had complete data at age 12 ( 54 boys and 50 girls) and 91 children at age 16 (44 boys and 47 girls).

\subsection{Procedure}

During a home visit at 15 months, the primary caregiver was interviewed about duration of breastfeeding. At 12 years of age, data was collected during a home visit after the parents had given informed active consent and the children had given verbal assent. During the home visit, children completed a questionnaire to assess alexithymia, EE and external eating behaviors. At 16 years of age, schools were asked to give informed permission for the project after the parents and the children had given active informed permission to approach the schools. Active consent was given by the school. Children completed questionnaires on EE and external eating behaviors during a classroom session.

\subsection{Measures}

\subsubsection{Duration of breastfeeding}

Breastfeeding information was collected at 15 months of age regarding the duration of breastfeeding in months. Mothers were asked "Did you breastfeed your baby?". If they said "no" this was recorded as "never breastfed." If they said "yes" they were asked "At what child age did you stop breastfeeding?". Maternal recall is found to be a valid and reliable estimate of breastfeeding initiation and duration, especially when the duration of breastfeeding is recalled after a short period (Li, Scanlon, \& Serdula, 2008). Duration of any breastfeeding was defined as the age at which breastfeeding stopped and was expressed as a continuous variable (in whole and half months).

\subsubsection{Alexithymia}

At 12 years, alexithymia "difficulty identifying feelings" and "difficulty describing feelings" were assessed, respectively, with 7 (e.g. "I have feelings that I can't quite identify"; Cronbach's alpha 0.71), and 5 (e.g. "It is difficult for me to find the right words for my feelings"; Cronbach's alpha 0.72) items of the Toronto Alexithymia Scale-20 (TAS20; Bagby, Parker, \& Taylor, 1994; Kooiman, Spinhoven, \& Trijsburg, 2002; adjusted for children by Rieffe, Oosterveld, \& Terwogt, 2006). The TAS-20 was chosen for the present study, as this questionnaire is designed to detect individuals who are alexithymic, and because the TAS-20 outperforms the LEAS (Levels of Emotional Awareness Scale; Lane, Quinlan, Schwartz, Walker, \& Zeitlin, 1990) when it comes to the assessment of the alexithymia aspect difficulty identifying feelings. Items were rated along a 3-point scale ranging from "disagree" (1) to "agree" (3). Scores for the scales were obtained by computing the mean of the items, with higher scores indicating more difficulty in identifying or describing emotions.

\subsubsection{Emotional and external eating}

At 12 years, EE and external eating were assessed, respectively, with 7 items ("If things go wrong do you get a desire for food?"; Cronbach's alpha 0.73), and 6 items ("Do you feel like eating whenever you see or smell good food?"; Cronbach's alpha .071) of the Dutch Eating Behavior Questionnaire for Children (DEBQ-C). The DEBQ-C is an age-adapted 20 -item version of the Dutch Eating Behavior Questionnaire (Van Strien, 2015), which is suitable for 7-12 year old children (Van Strien \& Oosterveld, 2008). The items had a three-point response format: (1) no - (5) yes.

At 16 years, EE and external eating were assessed with 6 (Cronbach's alpha 0.94), respectively 7 items (Cronbach's alpha 0.84 ) of the brief 20 -item version of the adult DEBQ (see also, Van Strien, Donker, et al., 2016; Paans et al., 2018). The items had a five-point response format: (1) never - (5) always. For both versions of the DEBQ, scale scores were obtained by computing the mean of the items. Higher scores reflect, respectively, more emotional and external eating.

\subsubsection{Parental level of education}

Paternal and maternal level of education was rated on a 7-point scale ranging from low (elementary school) to high (college degree or higher).

\subsection{Statistical analyses}

All analyses were carried out using SPSS version 23.0 (IBM Corporation, 2015). Moderated mediation (Fig. 1) was assessed with the PROCESS macro of SPSS version 23.0, developed by Hayes (2013, PROCESS macro, model 7). Moderated mediation in Model 7 is analysed with two regression equations (see Fig. 3, for the statistical diagram). In analysis 1) X, W, and the XW interaction are regressed on the mediator (M); In analysis 2) $\mathrm{M}$ and $\mathrm{X}$ are regressed on the dependent variable $(\mathrm{Y})$. All variables were centred before computing interaction terms (e.g. sex X duration of breastfeeding, see Fig. 3). Significance of 


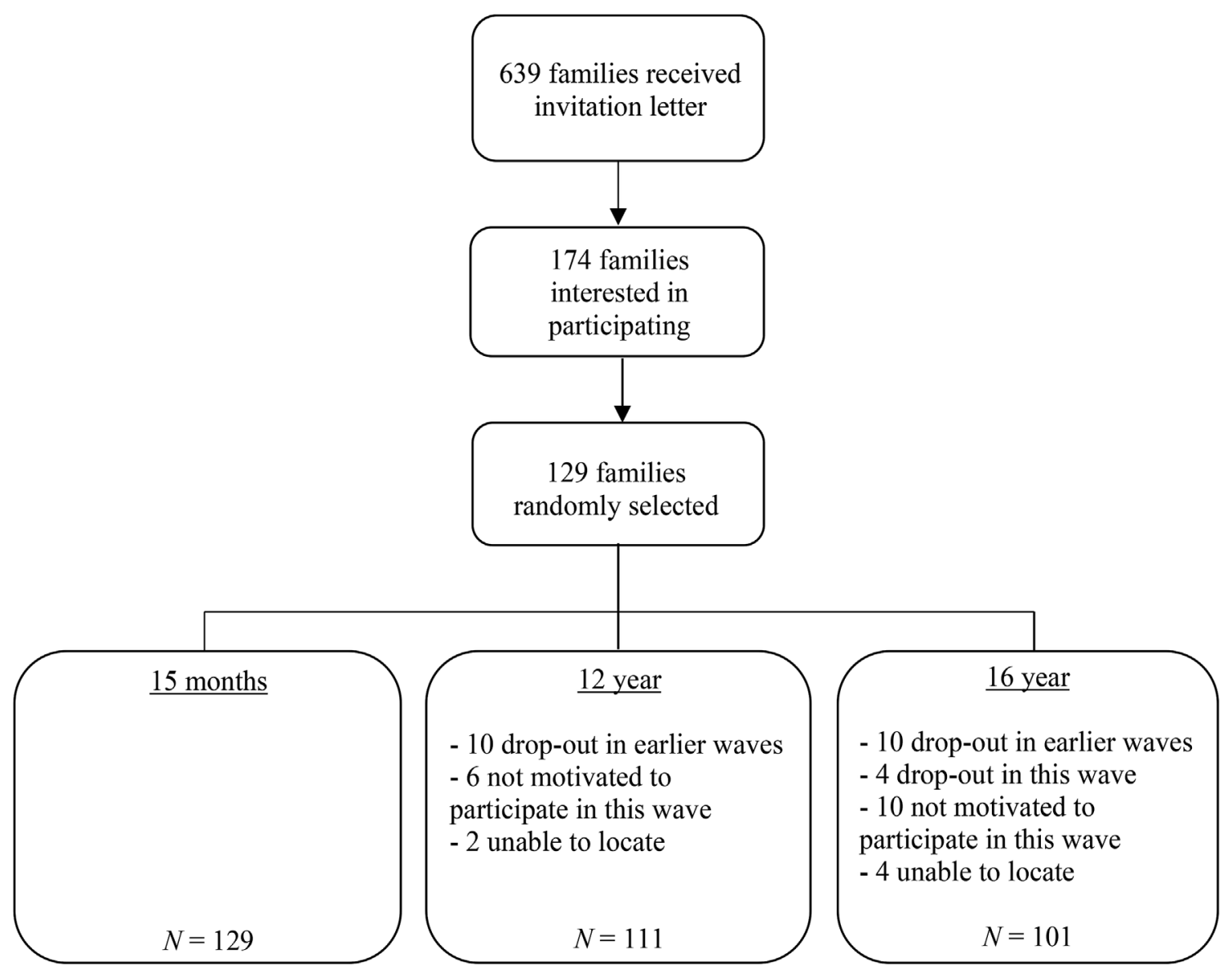

Fig. 2. Flow chart of the sample of the Nijmegen Longitudinal Study on Infant and Child Development.

moderated mediation was tested with Hayes' index of moderated mediation (Hayes, 2015). A test was deemed significant if the $95 \%$ bootstrap bias corrected confidence interval (CI) did not include zero. Bootstrapping with 5000 samples was used because "bootstrapping does not impose the assumption of normality of the sampling distribution" (Preacher \& Hayes, 2008, p. 880). In case the index of moderated mediation of our main research question was not significant at $95 \%$ bias corrected CI, we proceeded our analyses with a test of mediation only (PROCESS macro, model 4, not shown).

To assess the specificity of our moderated mediation model for the mediator alexithymia identifying feelings, we also tested the model for alexithymia describing emotions. In order to examine whether the above moderated mediation model is specific for EE, we assessed in an additional analysis the effects of breastfeeding duration through alexithymia on external eating.

Given that breastfeeding may be positively associated with parental

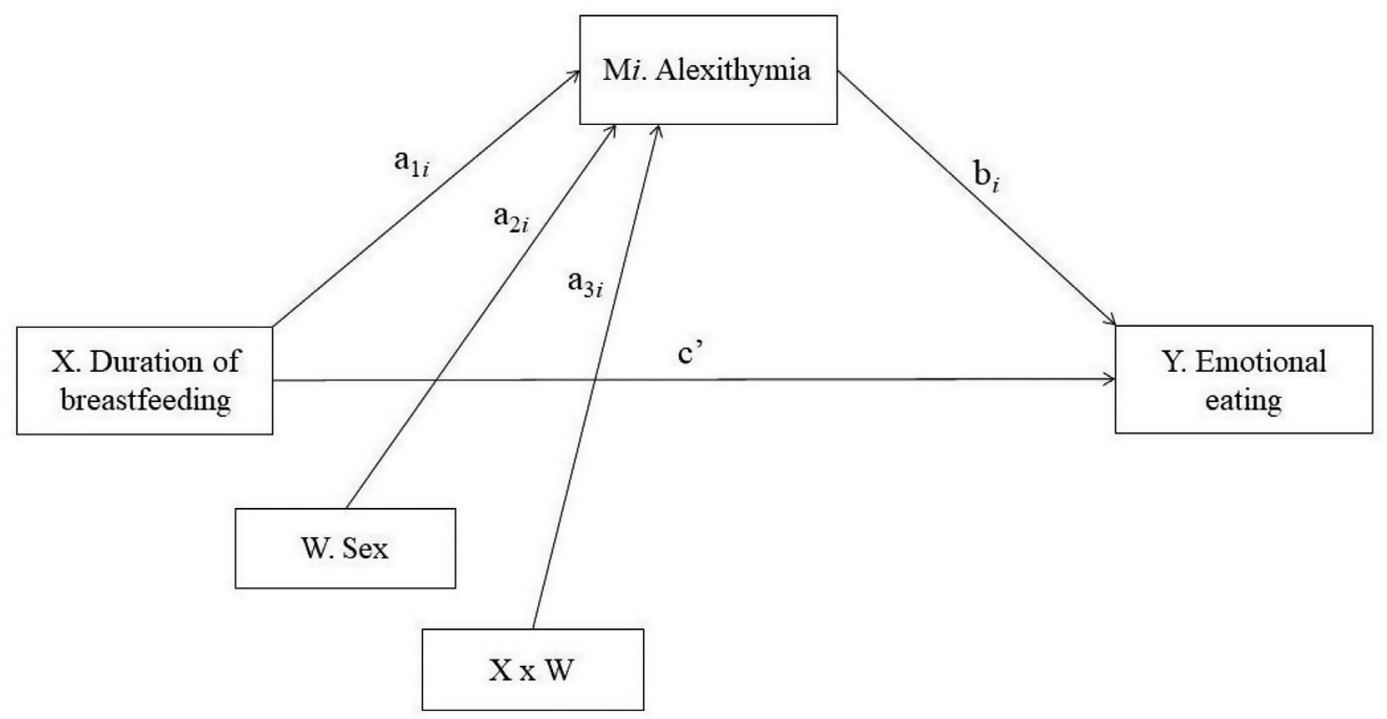

Fig. 3. Statistical diagram of moderated mediation: Conditional indirect effect of $\mathrm{X}$ on $\mathrm{Y}$ through $\mathrm{M}_{i=}\left(\mathrm{a}_{1 i}+\mathrm{a}_{3 i} \mathrm{~W}\right) \mathrm{bi}$ Direct effect of $\mathrm{X}$ on $\mathrm{Y}=c^{\prime}$. 
Table 1

Pearson correlations and mean and SD of the variables in the boys (below the diagonal) and the girls (above the diagonal).

\begin{tabular}{|c|c|c|c|c|c|c|c|c|c|}
\hline & & 1. & 2. & 3. & 4. & 5. & 6. & 7. & 8. \\
\hline \multicolumn{2}{|c|}{ 1. Breastfeeding duration } & - & .18 & .26 & .03 & .12 & .09 & .13 & .12 \\
\hline \multicolumn{2}{|c|}{ 2. Parental level of education } & $.50 * *$ & - & .02 & -.03 & $.30 *$ & $.27 *$ & $.42 *$ & .21 \\
\hline \multicolumn{2}{|c|}{ 3. Difficulty identifying feelings ${ }^{a}$} & -.25 & -.09 & - & $.39 *$ & $.36^{*}$ & .20 & -.09 & -.03 \\
\hline \multicolumn{2}{|c|}{ 4. Difficulty describing feelings ${ }^{a}$} & .07 & .08 & $.57 * *$ & - & .26 & .24 & -.17 & -.17 \\
\hline \multicolumn{2}{|c|}{ 5. Emotional eating - age 12} & -.10 & .01 & $.48^{* *}$ & .24 & - & $.57^{* *}$ & $.36^{*}$ & .09 \\
\hline \multicolumn{2}{|c|}{ 6. External eating - age 12} & .19 & .23 & $.28^{*}$ & .06 & $.60 * *$ & - & .25 & .22 \\
\hline \multicolumn{2}{|c|}{ 7. Emotional eating - age 16rowhead } & .06 & .05 & $.38^{*}$ & .25 & .28 & -.03 & - & $.69 * *$ \\
\hline \multicolumn{2}{|c|}{ 8. External eating - age 16} & .01 & -.12 & .21 & -.06 & .08 & .15 & $.29 *$ & - \\
\hline \multirow[t]{3}{*}{ Boys } & $M$ & 3.66 & 5.16 & 8.98 & 7.53 & 1.61 & 3.02 & 1.73 & 3.16 \\
\hline & $S D$ & 4.01 & 1.63 & 2.48 & 2.00 & 0.64 & 0.78 & 0.83 & 0.69 \\
\hline & $N$ & 67 & 67 & 59 & 59 & 54 & 54 & 47 & 47 \\
\hline \multirow[t]{3}{*}{ Girls } & $M$ & 3.67 & 4.75 & 9.76 & 7.92 & 1.80 & 3.07 & 2.53 & 3.08 \\
\hline & $S D$ & 3.45 & 1.82 & 1.94 & 2.05 & 0.67 & 0.76 & 1.00 & 0.75 \\
\hline & $N$ & 62 & 62 & 51 & 51 & 50 & 50 & 48 & 48 \\
\hline
\end{tabular}

*p $<0.05$ (two-tailed); **p $<0.001$ (two-tailed).

${ }^{\text {a }}$ Difficulty identifying feelings = alexithymia difficulty identifying feelings; difficulty describing feelings = alexithymia difficulty describing feelings.

level of education (Thulier \& Mercer, 2009), it was included as a covariate in the models. Because paternal and maternal level of education were highly interrelated $(r=0.69)$, the mean was calculated and included in the analyses. Because of high interrelations between EE and external eating $(r>0.55)$ we controlled in an additional analysis also for external eating (in the model with EE as dependent variable), and for $\mathrm{EE}$ in the additional model with external eating as dependent variable.

\section{Results}

\subsection{Preliminary analyses}

Table 1 shows means, standard deviations, and inter-correlations between the study variables for boys and girls separately. In both sexes, duration of breastfeeding was not significantly associated with alexithymia difficulty identifying and difficulty describing feelings, and also not with EE at 12 years or at 16 years of age. Furthermore, alexithymia difficulty identifying feelings was significantly associated with EE at 12 years for both boys and girls, and with EE at 16 years for boys only. Alexithymia difficulty identifying feelings and difficulty describing feelings were highly interrelated, and the same held true for EE and external eating at 12 years and 16 years. Only in girls, parental educational level was significantly associated with EE at 12 years and at 16 years of age.

\subsection{Moderated mediation with $E E$ at age 12 years as outcome variable}

Fig. 4 shows the results of the moderated mediation analysis (PROCESS, model 7) with sex as moderator variable (W) and alexithymia identifying feelings at 12 years as mediator $(\mathrm{M})$ in the link between breastfeeding duration $(\mathrm{X})$ and $\mathrm{EE}$ at 12 years of age $(\mathrm{Y})$. In the model with no confounders, the index of moderated mediation was significant ( $B=0.035$ ( $S E=0.017$ ), 95\% BootCI [0.0050, 0.0754]). Inspection of the conditional indirect effects revealed that there was a significant negative mediation effect of alexithymia difficulty identifying feelings for the boys ( $\mathrm{B}=-0.017$ ( $\mathrm{SE}=0.01$ ), 95\% BootCI $[-0.037,-0.0025])$, whereas there was no significant mediation effect of alexithymia difficulty identifying feelings for the girls ( $\mathrm{B}=0.018$ (SE $=0.014), 95 \%$ BootCI $[-0.0080,0.0487])$. The index of moderated mediation remained significant when controlling for the possible confounder parental educational level $(\mathrm{B}=0.036$, ( $\mathrm{SE}=0.018), 95 \%$ BootCI [0.0057, 0.0743]), and the same held true when we additionally controlled for external eating $(B=0.0253$ $(\mathrm{SE}=0.0129), 95 \%$ BootCI $[0.0045,0.0556])$. In all additional models, there was a $95 \%$ Boot CI significant negative mediation effect for alexithymia difficulty identifying feelings between duration of breastfeeding and EE in boys (see Fig. 5 for an illustration of the mediation effect in the boys).

\subsubsection{Specificity of the model at 12 years for $E E$}

In a subsequent analysis, it was tested whether the model is specific for EE by testing the model with external eating instead of EE as dependent variable $(\mathrm{Y})$. Though the index of moderated mediation was significant in the model with no confounders (B=0.0235 ( $\mathrm{SE}=0.0160$ ), 95\% BootCI [0.0025, 0.0665]), the index of moderated mediation was no longer significant in the full model when we controlled for parental educational level and $\mathrm{EE} \quad(\mathrm{B}=0.0011$ $(\mathrm{SE}=0.0077), 95 \%$ BootCI $[-0.0114,0.0214])$. This indicates that the model is specific for EE, because the index of moderated mediation remained significant in the initial model with $\mathrm{EE}$ as dependent variable, when additionally controlling for parental educational level and external eating in the full model.

\subsubsection{Specificity of the model at 12 years for alexithymia identifying feelings}

To test the specificity of the model for alexithymia identifying feelings, the initial model with EE as dependent variable $(\mathrm{Y})$ was also tested with the alexithymia scale difficulty describing feelings as mediator $(\mathrm{M})$. The index of moderated mediation was not significant $(\mathrm{B}=-0.0025$ (SE $=0.0100), 95 \%$ BootCI $[-0.0255,0.0150])$. This indicates that the model is indeed specific for alexithymia difficulty identifying feelings.

\subsection{Moderated mediation with EE at age 16 years as outcome variable}

A limitation of the previous moderated mediation analysis, is that the mediator and outcome had been simultaneously measured with one questionnaire at 12 years of age. In order to test the robustness of this causal chain as a causal chain in real time, we proceeded with re-conducting our analyses using the DEBQ scales on EE and external eating at 16 years of age $(n=91)$. The index of moderated mediation of the initial model (without confounders) was significant (B $=0.0242$ (Boot $\mathrm{SE}=0.0156)$, 95\% BootCI $[-0.0002 ; 0.0625])$. The index of moderated mediation analysis remained significant when we controlled for parental educational level $(B=0.0254$ (Boot $\mathrm{SE}=0.0158$ ), $95 \%$ BootCI [0.0007; 0.0651]). When we additionally controlled for external eating, the index of moderated mediation became borderline significant (90\% Boot CI): $\mathrm{B}=0.0207$ (Boot $\mathrm{SE}=0.0150$ ), 90\% BootCI [0.0015, 0.0536]. Inspection of the conditional indirect effects in the latter (full) model revealed that there was a $90 \%$ Boot CI borderline significant negative mediation effect of difficulty identifying feelings for the boys 


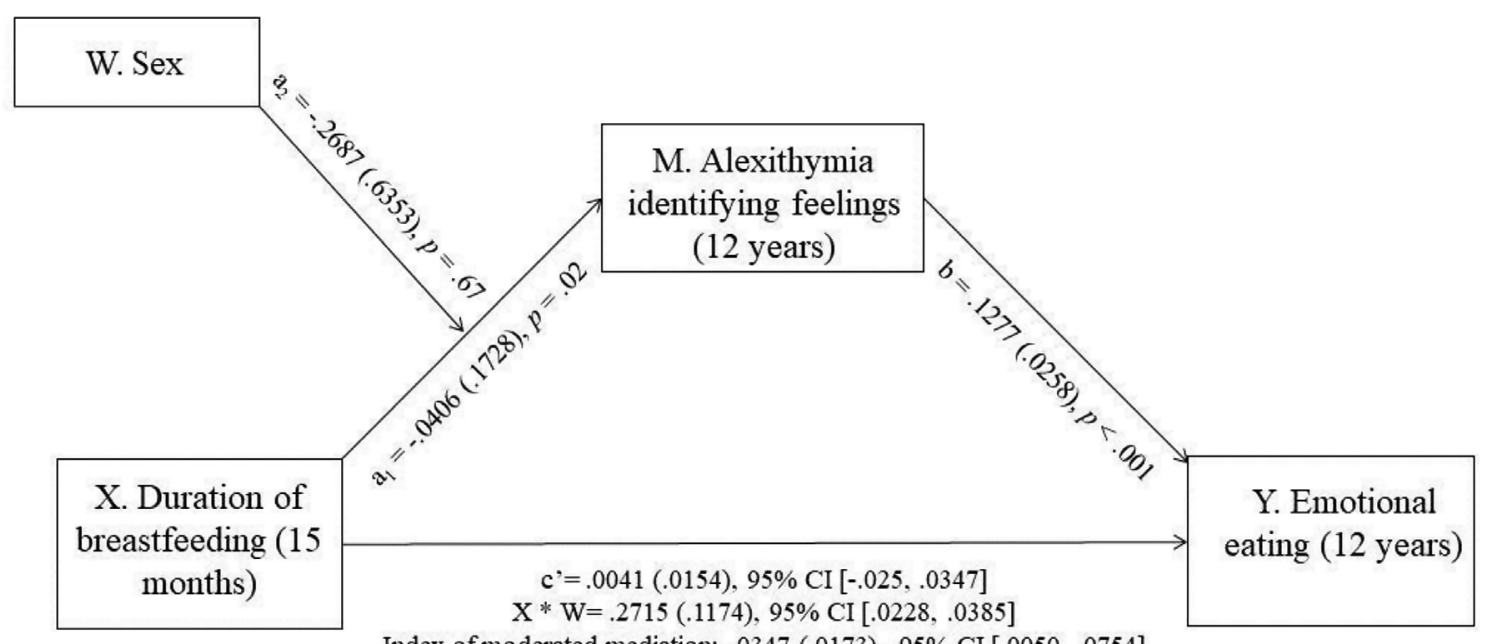

Fig. 4. Moderation of sex (W) on the mediation effect of alexithymia identifying feelings (M) between duration of breastfeeding (X) and EE (Y). There is a significant interaction effect of sex. Only in the boys a longer duration of breastfeeding is associated with a subsequent lower degree of alexithymia identifying feelings, resulting in lower EE (see Fig. 5).

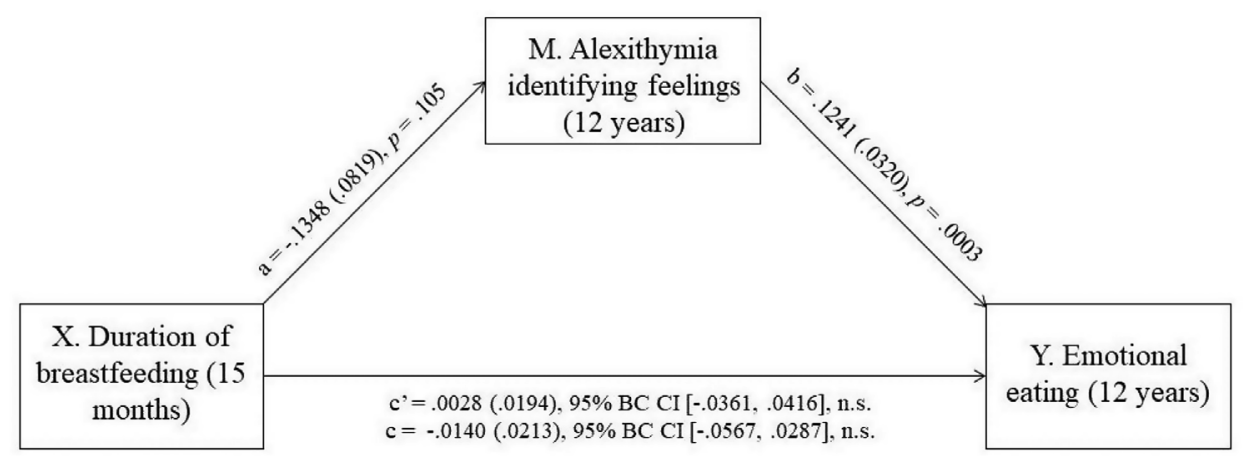

Fig. 5. Mediation effect of alexithymia identifying feelings $(\mathrm{M})$ between duration of breastfeeding $(\mathrm{X})$ and emotional eating $(\mathrm{Y})$ in the boys. The indirect effect of $\mathrm{X}$ on $\mathrm{Y}$ is significant $(\mathrm{B}=-0.0167)$ (Boot $\mathrm{SE}=0.0094)$, 95\% BootCI $[-0.0405,-0.0026]$.

$(\mathrm{B}=-0.0103(\mathrm{SE}=0.0076), 90 \%$ BootCI $[-0.0267,-0.0011])$, whereas there was no significant or borderline significant mediation effect of difficulty identifying feelings for the girls $(B=0.0104$ $(\mathrm{SE}=0.0110), 90 \%$ BootCI $[-0.0016,0.0349])$.

\subsubsection{Specificity of the model at age 16 years for $E E$}

In the model with external eating as dependent variable $(\mathrm{Y})$, the index of moderated mediation of the initial model (without confounders) was not significant, also not at $90 \%$ (BootCI: B $=0.0056$ (Boot SE $=0.0085$ ), 90\% BootCI [ $-0.0051,0.0232]$ ). This remained so when controlling for parental educational level and EE (not shown).

\subsubsection{Specificity of the model at age 16 years for alexithymia identifying} feelings

When testing the model with EE as dependent variable $(\mathrm{Y})$ and alexithymia difficulty describing feelings as mediator (M), the index of moderated mediation was also not significant at $95 \%$ BootCI or $90 \%$ BootCI (not shown).

\section{Discussion}

In this prospective study, we tested the hypothesis that breastfeeding duration is associated with subsequent emotional eating (EE) in adolescence, through its impact on alexithymia difficulty identifying feelings. We expected this hypothesis to be only true for boys, not girls. We thus expected that in the boys, a longer duration of breastfeeding would result in a lower degree of alexithymia identifying feelings, which, in turn, would result in a lower degree of EE. This hypothesis was significantly supported when investigating EE at 12 years, and borderline significantly supported when investigating EE at 16 years.

We found that breastfeeding duration was not related to alexithymia and subsequent EE in girls, which is consistent with some circumstantial evidence. This evidence suggests that breastfeeding may particularly protect boys against high degrees of alexithymia, through i) possible sex-specific effects of responsive parenting aspects of breastfeeding, or ii) oxytocin in breast milk (e.g., Browne et al., 2010; Rilling et al., 2014). Results from previous studies showing that the effects of exogenous administration of oxytocin on social interactions were only positive in men, can be explained by the possibility that oxytocin has an inverted U-shaped dose response function (Feng et al., 2015). Because women have higher baseline levels of oxytocin compared to men, raising the oxytocin level in women "might displace them at the right of the maximum, decreasing brain activity" (Feng et al., 2015, p. 761).

The possibility that oxytocin in breast milk shapes the phenotype of the offspring would fit in the Lactocrine Programming hypothesis (Bartol, Wiley, \& Bagnell, 2009; Miller, Wiley, Chen, Bagnell, \& Bartol, 2013). Accumulating evidence from animal studies suggests that maternal hormones, ingested through breast milk, affect offspring development in a sex-specific manner (Angelucci, Patacchioli, Chierichetti, \& Laureti, 1983; Casolini et al., 1997; Catalani et al., 2002; Hinde et al., 2014; Sullivan, Hinde, Mendoza, \& Capitanio, 2011). Though oxytocin in breast milk, to our knowledge, is not yet directly investigated in relation to offspring development, sex-specific relations have been observed between offspring behavior and the stress hormone cortisol in breast 
milk. For example, increased breast milk cortisol in monkeys was related to more confident temperament in male offspring (Sullivan et al., 2011), but to more nervous and less confident temperament in female offspring (Hinde et al., 2014). Additionally, increased milk cortisol was related to heightened Hypothalamic- Pituitary-Adrenal (HPA)-axis activity in female rodent offspring, but to decreased HPA-axis activity in male rodent offspring (Angelucci et al., 1983). Future research should investigate breast milk oxytocin to investigate sex-specific Lactocrine Programming effects on the offspring, including alexithymia.

It should be noted that, contradictory to our results, results from a large British study ( $\mathrm{r}$ 13500) suggested that breastfeeding had positive effects on emotional wellbeing, but in women only (Cable, Bartley, McMunn, \& Kelly, 2011). Emotional wellbeing was measured with items such as "Are you easily upset or frustrated?". A difference with our study was that the British study was conducted in two earlier cohorts (born in 1958 and 1970), where "most members of both cohorts were breastfed for $<1$ months or not breastfed at all" (Cable et al., 2011, p. 654). As a result, a dichotomous variable was created that indicated whether the child received breastfeeding for $\geq 1$ months (yes) or less than $<1$ month (no). In our study, $27 \%$ of the infants was breastfed for less than one month, and thus most infants were breastfed for longer. It can be questioned whether a brief period of breastfeeding, like in the British cohorts, is associated with responsive parenting and/ or sufficient exposure to breast milk hormones to have positive Lactocrine Programming effects for boys. For the girls, as they have higher baseline levels of oxytocin (Feng et al., 2015), this brief period may have been sufficiently short to show positive effects of oxytocin on later emotional well-being, but this hypothesis clearly needs testing.

The finding that the observed moderated mediation effect only held true for emotional eating, and not external eating (when adjusted for external eating, respectively emotional eating), adds to the literature showing that emotional and external eating, though highly interrelated ( $r>0.50$ ), should be conceived as distinct constructs with a different etiology and external validity (Van Strien, Herman, \& Verheijden, 2009; Van Strien, Herman, \& Anschutz, 2012; Van Strien, Konttinen, et al., 2016). The same holds true for our finding that our moderated mediation model was specific for alexithymia difficulty identifying feelings, similarly suggesting that alexithymia identifying and describing feelings are different constructs (see also, Tull, Medaglia, \& Roemer, 2005).

Of note, alexithymia was assessed with a child version of the TAS, and not with the performance based LEAS (Levels of Emotional Awareness Scale; Lane et al., 1990). The LEAS asks participants to describe their anticipated feelings and those of another person for each of 20 scenes (vignettes) (Lane et al., 1996). Though the LEAS seems to outperform the TAS when it comes to the assessment of the alexithymia aspect difficulty describing feelings, the TAS outperforms the LEAS when it comes to the assessment of the alexithymia aspect difficulty identifying feelings (Lane et al., 1996). Hence, for our present research question, the TAS is the instrument of choice. It is nevertheless recommended to replicate the present study using the LEAS, to assess whether our present results are robust for differences in the measurement of alexithymia.

A strength of this study is that we covered a long-term longitudinal time-span of 15 years with a low attrition rate. Moreover, similar results were obtained with the child version of the DEBQ (DEBQ-C) at 12 years, and the 20 -item adult version of the DEBQ at 16 years, which gives confidence in the robustness of the obtained results. A limitation of the current study is the use of self-reports. Participants may, for reason of social desirability, have disavowed their EE and external eating. Social desirability concerns, however, are not limited to studies that rely on self-reports. Social desirability may also affect behavior in direct tests of food intake, where specifically obese people may be motivated to avoid overeating, owing to the deviant status of obesity in our culture (Krantz, 1978). A problem with the measurement of alexithymia by self-report is that people who have difficulties to identify their feelings are asked to judge their own deficit in identifying feelings (Lane et al., 1990). Our measure of breastfeeding was collected from maternal recall through interviews at 15 months of infant age. Maternal recall is found to be a valid and reliable estimate of breastfeeding initiation and duration, especially when the duration of breastfeeding is recalled after a short period (Li et al., 2008). However, a limitation of our breastfeeding measure is that it does not differentiate between exclusive breastfeeding versus mixed feeding (a combination of breastfeeding and formula feeding). Some research has suggested that the benefits of breastfeeding might be more pronounced for exclusively breastfeeding mothers (Edwards, Thullen, Henson, Lee, \& Hans, 2015). As the amount of feedings not only predicts the amount of milk hormones ingested, but might also reflect responsive parenting styles, it is important for future research to not only take into account the duration of breastfeeding, but also the amount of feedings an infant receives per day.

Another strength of our study is that, as indicated by the distribution of parental and child measures at 15 months, the sample was representative of the Dutch population of families with young children (Van Bakel \& Riksen-Walraven, 2002a, 2002b). However, due to constraints in time and resources, our sample was relatively small, with 104 children having complete data at age 12, and 91 children having complete data at age 16. Future research should replicate our findings in larger samples.

Another factor for future research to take into account is whether infants are fed on-demand or at scheduled intervals. The composition of human milk, i.e., low protein and fat content, is indicative of a continuous, on-demand, feeding schedule, as is seen in a multitude of tribal cultures (Jansen, de Weerth, \& Riksen-Walraven, 2008). The practice of scheduling feeds has developed in Western civilization in order to accommodate working mothers, although on-demand breastfeeding is also seen. Breastfeeding on demand or at scheduled intervals may be particularly important when investigating the quality of parenting associated with these two types of breastfeeding. The 'Start Healthy Feeding Guidelines for Infants and Toddlers' of the American Dietetic Association recommend responsive parenting, recognizing and appropriately responding to infant hunger and satiety cues, including feeding on demand (Butte et al., 2004). Breastfeeding on demand might be especially related to responsive parenting, and subsequent less controlling and more responsive parental feeding and parenting practices later in childhood, when compared to breastfeeding at scheduled intervals, but this hypothesis needs further testing. This type of future research should also include attachment style, next to responsive parenting, as a mediator between breastfeeding practices and alexithymia. It has, for example, been found that breastfeeding duration predicts secure attachment at 24 months, irrespective of maternal sensitivity (Weaver et al., 2018), and also that features of anxious and avoidant insecure attachment styles are differentially related to separate facets of alexithymia (Oskis et al., 2013). Breastfeeding practices, including duration, amount and style of breastfeeding, could thus predict both responsive parenting and attachment style. Both, in turn, may independently predict alexithymia.

\section{Conclusion}

Breastfeeding in infancy may protect boys, but not girls, against emotional eating through its positive association with a better ability to identify feelings.

\section{Conflicts of interest}

Tatjana van Strien has a copyright and royalty interest in the Dutch Eating Behavior Questionnaire (DEBQ) and manual.

\section{Role of funding source}

This research was supported by a Sara van Dam Project Grant of the Royal Netherlands Academy of Arts and Sciences to Roseriet Beijers. 
Funding for Tatjana van Strien and Laura Winkens was provided by the European Union FP7 MooDFOOD Project 'Multi-country cOllaborative project on the rOle of Diet, FOod-related behavior, and Obesity in the prevention of Depression' (grant agreement no. 613598).

\section{Acknowledgement}

Tatjana van Strien wrote the first draft of the MS during her sabbatical-leave (autumn 2017) at the University of Valencia (Spain). She would like to thank Rosa María Baños Rivera for her hospitality and her roommate Marta Miragall Montilla for her helpful comments to an earlier version of the MS.

\section{Appendix A. Supplementary data}

Supplementary data to this article can be found online at https:// doi.org/10.1016/j.appet.2018.10.006.

\section{References}

Angelucci, L., Patacchioli, F. R., Chierichetti, C., \& Laureti, S. (1983). Perinatal motheroffspring pituitary-adrenal interrelationship in rats: Corticosterone in milk may affect adult life. Endocrinologia Experimentalis, 17, 191-205.

Bagby, R., Parker, J. D. A., \& Taylor, G. J. (1994). The twenty-item Toronto alexithymia scale: 1 . Item selection and cross validation of the factor structure. Journal of Psychosomatic Research, 38, 23-32.

Bartol, F. F., Wiley, A. A., \& Bagnell, C. A. (2009). Relaxin and maternal lactocrine programming of neonatal uterine development. Annals of the New York Academy of Sciences, 1160, 158-163.

Brown, A., \& Arnott, B. (2014). Breastfeeding duration and early parenting behavior: The importance of an infant-led, responsive style. PloS One, 9(2), e83893.

Browne, D. T., Odueyungbo, A., Thabane, L., Byrne, C., \& Smart, L. A. (2010). Parentingby-gender interactions in child psychopathology: Attempting to address inconsistencies with a Canadian national database. Child and Adolescent Psychiatry and Mental Health, 4(1), 5 .

Brown, A., \& Lee, M. (2012). Breastfeeding during the first year promotes satiety responsiveness in children aged 18-24 months. Pedriatic Obesity, 7(5), 382-390. https://doi.org/10.1111/j.2047-6310,2012.00071.x.

Bruch, H. (1973). Eating disorders. New York: Basic Books.

Butte, N., Cobb, K., Dwyer, J., Graney, L., Heird, W., \& Rickard, K. (2004). The start healthy feeding guidelines for infants and toddlers. Journal of the American Dietetic Association, 104, 442-454. https://doi.org/10.1016/j.jada.2004.01.027.

Cable, N., Bartley, M., McMunn, M., \& Kelly, Y. (2011). Gender differences in the effects of breastfeeding on adult psychological well-being. The European Journal of Public Health, 22(5), 653-658.

Carper, J. L., Fisher, J. O., \& Birch, L. L. (2000). Young girl's emerging dietary restraint and disinhibition are related to parental control in child feeding. Appetite, 35, 21-129.

Casolini, P., Cigliana, G., Alemà, G. S., Ruggieri, V., Angelucci, L., \& Catalani, A. (1997). Effect of increased maternal corticosterone during lactation on hippocampal corticosteroid receptors, stress response and learning in offspring in the early stages of life. Neuroscience, 79, 1005-1012.

Catalani, A., Casolini, P., Cigliana, G., Scaccianoce, S., Consoli, C., Cinque, C., et al. (2002). Maternal corticosterone influences behavior, stress response and corticosteroid receptors in the female rat. Pharmacology Biochemistry and Behavior, 73, 105-114.

Christov-Moore, L., Simpson, E. A., Coude, G., Grigaltyte, K., Iacoboni, M., \& Ferrari, P. F. (2014). Empathy: Gender effects in brain and behavior. Neuroscience \& Biobehavioral Reviews, 46, 604-627 http.//dx.doi.org/10.1016/j.neurobiorev.2014.09.001.

Disantis, K. I., Collins, B. N., Fisher, J. O., \& Davey, A. (2011). Do infants fed directly from the breast have improved appetite regulation an slower groth during early chandhood compared with infants fed from a bottle? International Journal of Behavioral Nutrition and Physical Activity, 8, 89. https://doi.org/10.1186/1479-5868-8-89.

Edwards, R. C., Thullen, M. J., Henson, L. G., Lee, H., \& Hans, S. L. (2015). The Association of breastfeeding initiation with sensitivity, cognitive stimulation, and efficacy among young mothers: A propensity score matching approach. Breastfeeding Medicine, 10, 13-19. https://doi.org/10.1089/bfm.2014.0123.

Escobar, R. S., O'Donnell, K. A., Colalillo, S., Pawlby, S., Steiner, M., Meaney, M. J., et al. (2014). Better quality of mother-child interaction at 4 years of age decreases emotional overeating in IUGR girls. Appetite, 81, 337-342. https://doi.org/10.1016/j appet.2014.06.107.

Feng, C., Hackett, P. D., DeMarco, A. C., Chen, X., Stair, S., Haroon, E., ... Rilling, J. K. (2015). Oxytocin and vasopressin effects on the neural response to social cooperation are modulated by sex in humans. Brain Imaging and Behavior, 9(4), 754-764.

Garner, D. M. (1991). Eating disorder inventory-2 manual. Odessa, FL: Psychological Assessment Resources.

Gold, P. W., \& Chrousos, G. P. (2002). Organisation of the stress system and its dysregulation in melancholic and typical depression. High vs low CRH/NE states. Molecular Psychiatry, 7, 254-275.

Grynberg, D., Chang, B., Corneille, O., Maurage, P., Vermeulen, N., Berthoz, S., et al.
(2012). Alexithymia and the processing of emotional facial expressions (EFEs): Systematic review, unanswered questions and further perspectives. PloS One, 7(8), e42429. https://doi.org/10.1371/journal.pone.0042429.

Hayes, A. F. (2013). Introduction to mediation, moderation, and conditional process analysis: A regression-based approach. New York, London: The Guilford Press.

Hayes, A. F. (2015). An index and test of linear moderated mediation. Multivariate Behavioral Research, 50(1), 1-22.

Hinde, K., Skibiel, A. L., Foster, A. B., Del Rosso, L., Mendoza, S. P., \& Capitanio, J. P. (2014). Cortisol in mother's milk across lactation reflects maternal life history and predicts infant temperament. Behavioral Ecology, 26(1), $269=281$.

IBM Corp. Released (2015). IBM SPSS Statistics for Windows, version 23.0. Armonk, NY: IBM Corp.

Jansen, J., de Weerth, C., \& Riksen-Walraven, J. M. (2008). Breastfeeding and the motherinfant relationship - a review. Developmental Review, 28, 503-521. https://doi.org/10. 1016/j.dr.2008.07.001.

Jansen, E., Mallan, K. M., Byrne, R., Daniels, L. A., \& Nicholson, J. M. (2016). Breastfeeding duration and authoritative feeding practices in first-time mothers. Journal of Human Lactation, 32(3), 498-506. https://doi.org/10.1177/ 0890334415618669.

Klump, K. L., Racine, S. E., Hildebrandt, B., Burt, S. A., Neale, M., Sisk, C. L., et al. (2014) Ovarian hormone influences on dysregulated eating: A comparison of associations in women with versus without binge episodes. Clinical Psychological Science, 2(4), 545-559. https://doi.org/10.1177/2167702614521794.

Koenders, P., \& Van Strien, T. (2011). Emotional eating rather than lifestyle behavior drives weight gain in a prospective study in 1562 employees. Journal of Occupational and Environmental Medicine, 53, 1287-1293.

Kooiman, C. G., Spinhoven, P., \& Trijsburg, R. W. (2002). The assessment of alexithymia: A critical review of the literature and a psychometric study of the alexithymia scale20. Journal of Psychosomatic Research, 53, 1083-1090.

Krantz, D. S. (1978). The social context of obesity research. Another perspective on its place in the field of social psychology. Personality and Social Psychology Bulletin, 4, $177-184$.

Krol, K. M., Monakhov, M., San Lai, P., Ebstein, R. P., \& Grossmann, T. (2015b). Genetic variation in CD38 and breastfeeding experience interact to impact infants' attention to social eye cues. Proceedings of the National Academy of Sciences, 112(39), E5434-E5442. https://doi.org/10.1073/pnas.1506352112.

Krol, K. M., Rajhans, P., Missina, M., \& Grossmann, T. (2015a). Duration of exclusive breastfeeding is associated with differences in infants' brain responses to emotional body expressions. Frontiers in Behavioral Neuroscience, 8. https://doi.org/10.3389/ fnheh.2014.00459.

Lane, R. D., Quinlan, D. M., Schwartz, G. E., Walker, P. A., \& Zeitlin, S. B. (1990). The levels of emotional awareness scale: A cognitive-developmental measure of emotion. Journal of Personality Assessment, 55, 124-134.

Lane, R. D., Sechrest, L., Reidel, R. G., Weldon, V., Kaszniak, A. W., \& Schwartz, G. E. (1996). Impaired verbal and nonverbal emotion recognition in alexithymia. Psychosomatic Medicine, 58, 203-210.

Larsen, J. K., van Strien, T., Eisinga, R., \& Engels, R. C. (2006). Gender differences in the association between alexithymia and emotional eating in obese individuals. Journal of Psychosomatic Research, 60(3), 237-243.

Levant, R. F., Hall, R. J., Williams, C. M., \& Hasan, N. T. (2009). Gender differences in alexithymia. Psychology of Men and Masculinity, 10(3), 190-203.

Li, R., Scanlon, K. S., \& Serdula, M. K. (2008). The validity and reliability of maternal recall of breastfeeding practice. Nutrition Reviews, 63(4), 103-110. https://doi.org/ 10.1111/j.1753-4887.2005.tb00128.x.

Luminet, O., Grynberg, D., Ruzette, N., \& Mikolajczak, M. (2011). Personality-dependent effects of oxytocin: Greater social benefits for high alexithymia scorers. Biological Psychology, 87, 401-406.

Michels, N., Sioen, I., Ruigem, J., \& De Henauw, S. (2017). Children's psychological stress and emotional eating: A role for leptin? International Journal of Eating Disorders, 50, 471-480.

Miller, D. J., Wiley, A. A., Chen, J. C., Bagnell, C. A., \& Bartol, F. F. (2013). Nursing for 48 hours from birth supports porcine uterine gland development and endometrial cell compartment-specific gene expression. Biology of Reproduction, 88(1), 1-10.

Nommsen, L. A., Lovelady, C. A., Heinig, M. J., Lönnerdal, B., \& Dewey, K. G. (1991) Determinants of energy, protein, lipid and lactose concentrations in human milk during the first 12 months of lactation: The DARLING study. American Journal of Clinical Nutrition, 53, 457-465.

Oskis, A., Clow, A., Hucklebridge, F., Bifulco, A., Jacobs, C., \& Loveday, C. (2013). Understanding alexithymia in female adolescents: The role of attachment style. Personality and Individual Differences, 54, 97-102. https://doi.org/10.1016/j.paid. 2012.08.023.

Paans, N. P. G., Bot, M., Van Strien, T., Brouwer, I. A., Visser, M., \& Penninx, B. W. J. H. (2018). Eating styles in major depressive disorder: Results from a large-scale study. Journal of Psychiatric Research, 97, 38-46. https://doi.org/10.1016/j.jpsychires.2017. 11.003.

Preacher, K. J., \& Hayes, A. (2008). Asymptotic and resampling strategies for assessing and comparing indirect effects in multiple mediator models. Behavior Research Methods, 40, 879-891.

Reyes, M., Hoyos, V., Martinez, S. M., Lozoff, B., Castillo, M., Burrows, R., et al. (2014) Satiety responsiveness and eating behavior among Chilean adolescents and the role of breastfeeding. International Journal of Obesity, 38(4), 552-557. https://doi.org/10. 1038/ijo.2013.191.

Rieffe, C., Oosterveld, P., \& Terwogt, M. M. (2006). An alexithymia questionnaire for children: Factorial and concurrent validation results. Personality and Individual Differences, 40(1), 123-133.

Rilling, J. K., DeMarco, A. C., Hacket, P. D., Chen, X., Gautam, P., Stair, S., et al. (2014) 
Sex differences in the neural and behavioral response to intranasal oxytocin and vasopressin during human social interaction. Psychoneuroendrology, 39, 237-248.

Schwartz, M. W., Woods, S. C., Porte, D., Jr., Seeley, R. J., \& Baskin, D. G. (2000). Central nervous system control of food intake. Nature, 404(6778), 661.

Shafai, T., Mustafa, M., Mulari, J., \& Curtis, A. (2017). Impact of infant feeding methods on the development of autism spectrum disorder. In M. Fitzgerald (Ed.). Autismparadigms, recent research and clinical applications (pp. 80). InTech. https://doi.org/ $10.5772 / 67624$.

Shloim, N., Shafiq, I., Blundell-Birtill, P., \& Hetherington, M. M. (2018). Infant hunger and satiety cues during the first two years of life: Developmental changes within meal signaling. Appetite, 128, 303-310. https://doi.org/10.1016/j.appet.2018.05.144.

Shloim, N., Vereijken, C. M. J. L., Blundell, P., \& Hetherington, M. M. (2017). Looking for cues-infant communication of hunger and satiation during milk feeding. Appetite, 107, 74-82.

Snoek, H. M., Engels, R. C. M. E., Janssens, J. M. A. M., \& Van Strien, T. (2007). Parental behaviour and adolescents'emotional eating. Appetite, 49, 223-230.

Sullivan, E. C., Hinde, K., Mendoza, S. P., \& Capitanio, J. P. (2011). Cortisol concentrations in the milk of rhesus monkey mothers are associated with confident temperament in sons, but not daughters. Developmental Psychobiology, 53, 96-104.

Taveras, E. M., Scanlon, K. S., Birch, L., Rifas-Shiman, S. L., Rich-Edwards, J. W., \& Gillman, M. W. (2004). Association of breastfeeding with maternal control of infant feeding at age 1 year. Pedriatrics, 114(5), https://doi.org/10.1542/peds.2004-0801 e577-583.

Thorberg, F. A., Young, R. M. D., Sullivan, K. A., \& Lyvers, M. (2011). Parental bonding and alexithymia: A meta-analysis. European Psychiatry, 26, 187-193. https://doi.org/ 10.1016/j.eurpsy.2010.09.010.

Thulier, D., \& Mercer, J. (2009). Variables associated with breastfeeding duration. Journal of Obstetric, Gynecologic, and Neonatal Nursing, 38, 259-268.

Tull, M. T., Medaglia, E., \& Roemer, L. (2005). An investigation of the construct validity of the 20-Item Toronto Alexithymia Scale through the use of a verbalization task. Journal of Psychosomatic Research, 59(2), 77-84.

Uvnas-Moberg, K. (1998). Oxytocin may mediate the benefits of positive social interaction and emotions. Psychoneuroendocrinology, 23, 819-835. https://doi.org/10.1016/ s0306-4530(98)00056-0.

Van Bakel, H. J., \& Riksen-Walraven, J. M. (2002a). Parenting and development of one-year-olds: Links with parental, contextual, and child characteristics. Child Development, 73(1), 256-273.

Van Bakel, H. J., \& Riksen-Walraven, J. M. (2002b). Quality of infant-parent attachment as reflected in infant interactive behaviour during instructional tasks. Journal of Child Psychology and Psychiatry, 43(3), 387-394.

Van Stien, T., \& Bazelier, F. G. (2007). Perceived parental control of food intake is related to external, restrained and emotional eating in 7-12-year-old boys and girls. Appetite,
49, 618-625.

Van Strien, T. (2015). The Dutch eating behavior questionnaire. Amsterdam: Hogrefe.

Van Strien, T., Donker, M. H., \& Ouwens, M. A. (2016). Is desire to eat in response to positive emotions an 'obese'eating style: Is Kummerspeck for some people a misnomer? Appetite, 100, 225-235.

Van Strien, T., Engels, R. C. M. E., Van Leeuwe, J., \& Snoek, H. M. (2005). The Stice model of overeating: Tests in clinical and non-clinical samples. Appetite, 45(3), 205-213.

Van Strien, T., Herman, C. P., \& Anschutz, D. J. (2012). The predictive validity of the DEBQ-external eating scale for eating in response to food commercials while watching television. International Journal of Eating Disorders, 45, 257-262.

Van Strien, T., Herman, C. P., \& Verheijden, M. W. (2009). Eating style, overeating and overweight in a representative Dutch sample: Does external eating play a role? Appetite, 52, 380-387.

Van Strien, T., Herman, C. P., \& Verheijden, M. W. (2012). Eating style, overeating and weight gain: A prospective 2-year follow-up study in a representative Dutch sample. Appetite, 59, 782-789.

Van Strien, T., Konttinen, H., Homberg, J. R., Engels, R. C. M. E., \& Winkens, L. H. H. (2016). Emotional eating as a mediator between depression and weight gain. Appetite, $100,216-224$.

Van Strien, T., \& Oosterveld, P. (2008). The children's DEBQ for assessment of restrained, emotional, and external eating in 7-to 12-year-old children. International Journal of Eating Disorders, 41(1), 72-81.

Van Strien, T., \& Ouwens, M. A. (2007). Effects of distress, alexithymia and impulsivity on eating. Eating Behaviors, 8(2), 251-257.

Van Strien, T., Van Niekerk, R., \& Ouwens, M. A. (2009). Perceived parental food controlling practices are related to obesogenic or leptogenic home environments. Appetite, 53, 151-154.

Ventura, A. K. (2017). Associations between breastfeeding and maternal responsiveness: A systematic review of the literature. Advances in Nutrition, 8(3), 495-510. https:// doi.org/10.3945/an.116.014753.

Vittengl, J. R. (2018). Mediation of the bidirectional relations between obesity and depression among women. Psychiatry Research, 264, 254-259.

Wardle, J. (1990). Conditioning processes and cue exposure in the modification of excessive eating. Addictive Behaviors, 15, 387-393.

Weaver, J. M., Schofield, T. J., \& Papp, L. M. (2018). Breastfeeding duration predicts greater maternal sensitivity over the next decade. Developmental Psychology, 54, 220-227. https://doi.org/10.1037/dev0000425.

Woo, J. G., \& Martin, L. J. (2015). Does breastfeeding protect against childhood obesity? Moving beyond observational evidence. Current Obesity Reports, 4, 207-216.

Zhu, H., Luo, X., Cai, T., Li, Z., \& Liu, W. (2014). Self-control and parental control mediate the relationship between negative emotions and emotional eating among adolescents. Appetite, 82, 202-207. https://doi.org/10.1016/j.appet.2014.06.106. 